\title{
Observations on core turbulence transitions in ASDEX Upgrade using Doppler reflectometry
}

\author{
G.D.Conway, C.Angioni, R.Dux, F.Ryter, A.G.Peeters, \\ J.Schirmer, C.Troester, CFN Reflectometry Group* and \\ ASDEX Upgrade Team \\ Max-Planck-Institut für Plasmaphysik, EURATOM-Association IPP, Garching, \\ D-85748, Germany \\ * EURATOM-IST Association, Centro Fusão Nuclear, Lisboa, Portugal
}

\begin{abstract}
In Doppler reflectometry the antenna tilt angle $\theta_{\mathrm{t}}$ induces a Doppler frequency shift $f_{\mathrm{D}}=u_{\perp} 2 \sin \theta_{\mathrm{t}} / \lambda_{\mathrm{o}}$ in the measured spectrum which is directly proportional to the rotation velocity $u_{\perp}=v_{E \times B}+v_{\mathrm{ph}}$ of the turbulence moving in the plasma. Measurements in ohmic ASDEX Upgrade core plasmas show $u_{\perp}$ of the order of $1-2 \mathrm{~km} \mathrm{~s}^{-1}$ and reversing direction with increasing collisionality. Numerical simulations of the turbulence phase velocity $v_{\text {ph }}$ using the GS2 linear gyrokinetic code reveal a change in the dominant core turbulence from ITG to TEM with decreasing collisionality. The transition coincides with the $u_{\perp}$ reversal. Extracting the $E \times B$ velocity from the measured $u_{\perp}$ and the simulation $v_{\mathrm{ph}}$ indicates that the core radial electric field reverses sign with the turbulence. Using the radial force balance equation $v_{E \times B}=v_{\perp}-v^{*}$ and measured diamagnetic velocities $v^{*}$ gives a perpendicular fluid velocity $v_{\perp \mathrm{i}}$ reversing from $\sim 5$ to $7.5 \mathrm{~km} \mathrm{~s}^{-1}$ (ion direction) at low collisionality to $-0.7 \mathrm{~km} \mathrm{~s}^{-1}$ (electron direction) at high collisionality. Neoclassical predictions using the NEOART and NCLASS codes give poloidal Deuterium fluid velocities too small by factor of ten. Toroidal ion fluid velocities would need to be significant $\left(>30 \mathrm{~km} \mathrm{~s}^{-1}\right)$ at low $\nu^{*}$ to account for the difference. A clear ITG to TEM transition in the core turbulence has also been demonstrated using on-axis electron cyclotron heating to perturb the collisionality.
\end{abstract}

Submitted to: Nucl. Fusion

PACS numbers: 52.55.Fa, 52.35.Ra, 52.70.Gw 


\section{Introduction}

Doppler reflectometry is a potentially powerful diagnostic technique for measuring the plasma $E \times B$ rotation velocity profile in tokamaks or stellarators. Using a microwave reflectometer for density fluctuation measurements but with the launch and receive antennas deliberately tilted, so as to create an angle between the launched beam and the normal to the plasma cutoff layer, results in a hybrid diagnostic with the wavenumber sensitivity of coherent scattering, $k_{\perp}=2 k_{\mathrm{o}} \sin \theta_{\mathrm{t}}$ (perpendicular density fluctuation wavenumber defined by Bragg selection), and the excellent radial localization of reflectometry $[1,2]$. If the density fluctuations at the cutoff layer are moving then the measured fluctuation spectrum is Doppler frequency shifted

$$
f_{\mathrm{D}}=u_{\perp} k_{\perp} / 2 \pi=u_{\perp} 2 \sin \left(\theta_{\mathrm{t}}\right) / \lambda_{\mathrm{o}} .
$$

The Doppler shift $f_{\mathrm{D}}$ is directly proportional to the tilt angle $\theta_{\mathrm{t}}$ and the perpendicular (to $B$ ) rotation velocity of the turbulence moving in the plasma,

$$
u_{\perp}=v_{E \times B}+v_{\mathrm{ph}} .
$$

In the plasma edge the $E \times B$ velocity $v_{E \times B}$ is generally much larger than the phase velocity $v_{\mathrm{ph}}$ of the dominant drift-wave turbulence, which allows the radial electric field $E_{\mathrm{r}}=-v_{E \times B} B$ to be extracted directly from the measured $u_{\perp}$ velocity. (Note that $u_{\perp}$ is not the fluid velocity). However, in the plasma core, i.e. inside the density pedestal radius, the density turbulence is expected to change form and become more ITG (ion temperature gradient) and TEM (trapped electron mode) like. In this case the turbulence phase velocity may no longer be insignificant - which complicates the extraction of the $E_{\mathrm{r}}$. Unfortunately the separation of the $v_{E \times B}$ and $v_{\mathrm{ph}}$ components is not straightforward. For example, experimentally one might attempt a differential measurement by reversing either the $v_{E \times B}$ and $v_{\mathrm{ph}}$ directions independently by switching the toroidal $B_{\phi}$ or poloidal $B_{\theta}$ magnetic field (via $I_{\mathrm{p}}$ ). But this fails since the turbulence generally goes with the diamagnetic velocity $v^{*}$ which also reverses with $B$. Nevertheless, there is one possible experimental route. Theory and numerical simulations together with experimental observations indicate that the dominant core turbulence may switch between TEM and ITG - with a consequent reversal in the phase velocity direction with plasma conditions, e.g. most notably the average plasma density [3]. In addition to aiding the diagnostic development, an experimental confirmation of such a transition would be of high interest for validating turbulence models. This is the purpose of the experiments presented here.

Two approaches have been investigated. The first is to use a series of ohmic discharges with varying line average density to mimic conditions previously found to indicate either core ITG or TEM dominance, such as density peaking/flattening and heat conductivity $\chi_{\mathrm{e}}$ effects, and commensurate with predictions from numerical turbulence simulations [3, 4]. Using ohmic heated discharges also has the advantage that the toroidal fluid rotation is expected to be small - compared to neutral beam injection (NBI) heated scenarios for example - so that the $E \times B$ velocity should be comparable 


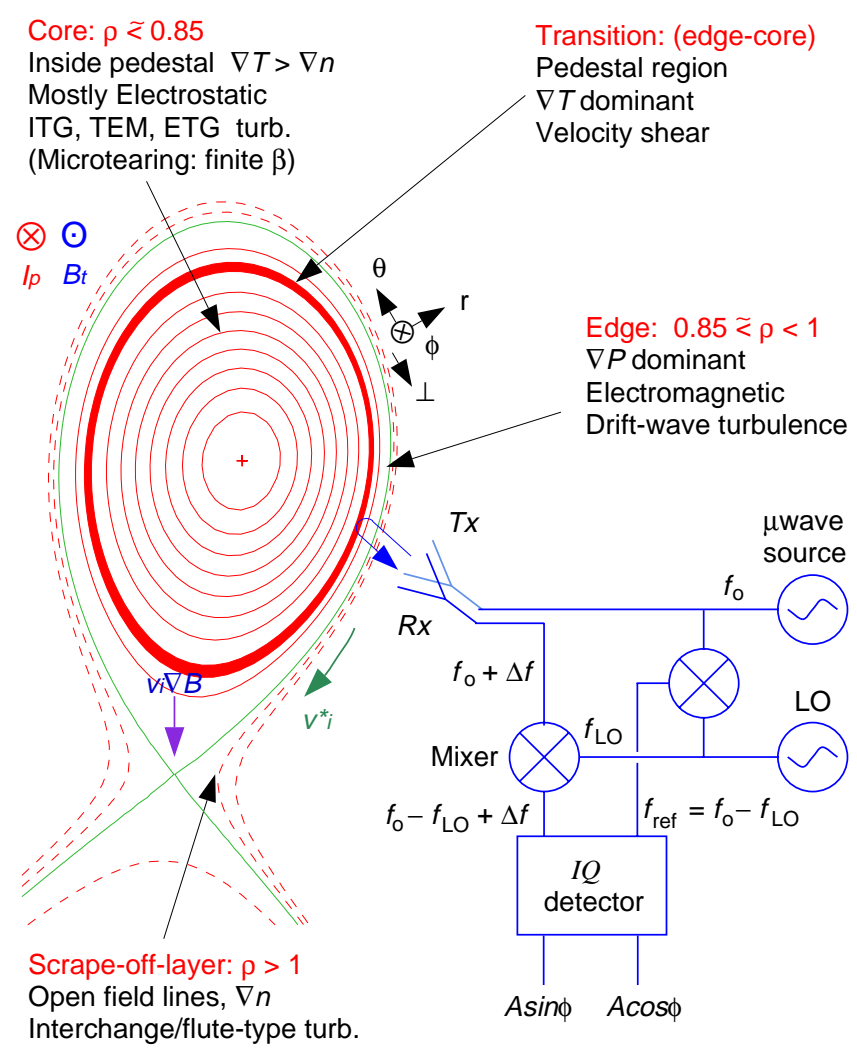

Figure 1. Poloidal cross section of a typical diverted AUG discharge with schematic of Doppler reflectometer.

in magnitude to expected phase velocities - which in turn should help to maximise the observation of any transitions in the $u_{\perp}$. Measurements indeed show the core $u_{\perp}$ reversing direction with increasing collisionallity which is reminiscent of the $E_{\mathrm{r}}$ reversals observed in the CHS [5] and LHD stellarators [6]. However, the stellarator results were attributed to purely neoclassical effects. Therefore we compare our measurements with computed turbulence phase velocities from the GS2 linear gyrokinetic turbulence code, as well as with measured diamagnetic velocities and predicted poloidal fluid velocities from neoclassical theory.

The second approach to discriminate between $v_{\mathrm{ph}}$ and $v_{E \times B}$ uses moderate levels of $140 \mathrm{GHz}$ electron cyclotron resonance heating (ECRH) power applied on-axis at densities close to the predicted transition so as to perturb the collisionallity and hence provoke controlled turbulence transitions. Although changes in the direction of Doppler shifts are again observed the results show some unexpected anomalies.

\section{Doppler diagnostics}

Figure 1 shows a poloidal cross section of the flux surfaces of a typical lower single null diverted ASDEX Upgrade (AUG) plasma, together with a schematic of a Doppler reflectometer diagnostic. For the experiments described here two microwave 


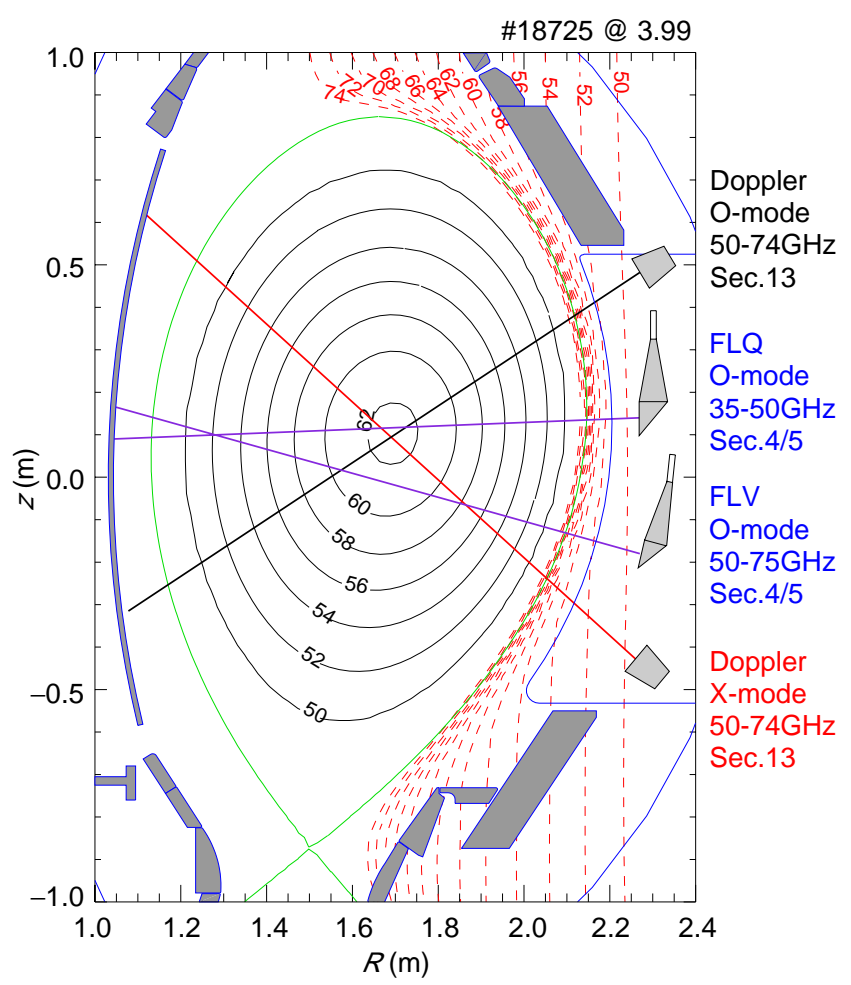

Figure 2. Poloidal cross section showing antenna lines of sight and O-mode (black $\longrightarrow$ ) and X-mode (red - - - ) cutoff layers for V-band frequencies in GHz.

reflectometer systems are used. The first system is a dedicated heterodyne Doppler reflectometer consisting of two identical channels with variable launch frequencies, $f_{\mathrm{o}}=c / \lambda_{\mathrm{o}}$, between $50-75 \mathrm{GHz}$ (V-band). The channels can be configured to operate together (for radial correlation measurements) or individually in either O-mode or Xmode polarization by switching between launch and receive antenna pairs positioned above and below the tokamak outboard mid-plane. The antennas currently have fixed poloidal inclinations, although the actual angle of incidence with the cutoff layers can be varied via the plasma shape. Typically the launch frequencies are stepped through the full V-band in 100 to $400 \mathrm{~ms}$ to provide radial profiles. Depending on the plasma density and toroidal magnetic field the cutoff layers (measurement positions) can be scanned from the scrape-off-layer (SOL) to the density pedestal in X-mode, and from the plasma edge to approximately mid-radius on the low field side in O-mode. In-phase $(I=A \cos \phi)$ and Quadrature $(Q=A \sin \phi)$ fluctuation signals (allowing phase and amplitude separation) are sampled at $20 \mathrm{MHz}$ with a Doppler frequency discrimination upto $7 \mathrm{MHz}$ (defined by anti-aliasing filters). Full details of the diagnostic can be found in reference [2] together with illustrative examples of $u_{\perp}$ radial profiles from various AUG plasma scenarios.

The second system is the recently upgraded fluctuation monitor reflectometers $[7,8]$. There are two separate channels, one (designated FLQ) operating in the microwave Qband $(35-50 \mathrm{GHz})$ and one (designated FLV) in the V-band $(50-75 \mathrm{GHz})$. At 
the time of the measurements the two launch frequencies could be set within their respective bands on a shot-by-shot basis. Each reflectometer channel uses a single monostatic antenna for both launching and receiving with an in-vessel directional coupler for signal separation. Detection is again heterodyne with $I$ and $Q$ signals collected at a fixed sample frequency of $500 \mathrm{kHz}$. These channels were not designed for Doppler measurements but happen to provide accidentally useful measurements in certain discharge conditions due to the alignment of the antennas. In a medium sized device, such as ASDEX Upgrade, it is a well known problem that it is almost impossible to design a reflectometer (that has non-steerable antennas) to be normally incident on the plasma cutoff layer for all plasma conditions. In this respect, all fluctuation reflectometers are potential Doppler reflectometers! Figure 2 shows the poloidal positions of the antennas for the four channels with their fixed vacuum lines of sight. Note the two sets of antennas are in different toroidal sectors of the tokamak. Also shown are the cutoff layers (cold plasma approximation with normal incidence) for O-mode (black solid) and X-mode (red dashed) at selected frequencies across the V-band for the typical medium density $\left(\bar{n}_{\mathrm{e}}=4 \times 10^{19} \mathrm{~m}^{-3}\right)$ ohmic discharge \#18725.

\section{Turbulence overview}

Also marked on the poloidal cross section in figure 1 are the various regions of the tokamak plasma with their respective turbulence characteristics. In the absence of an upto-date comprehensive experimental review of tokamak turbulence (c.f. Liewer 1985 [9] or Wootton 1990 [10]) a brief overview of the salient features is given here.

The outermost plasma region is the scrape-off-layer (SOL) where the open field lines connect to the divertor (normalized poloidal flux surface coordinate $\rho_{\text {pol }}>1$ ). Here the turbulence is predominately low frequency, long wavelength $\left(k_{\perp} \rho_{\mathrm{s}} \leq 0.1\right)$ interchange or flute mode type, driven by the density gradient and field line curvature $[11,12]$. The dispersion relation $v_{\mathrm{ph}}=\omega / k$ should be linear with phase velocities upto $1 \mathrm{~km} \mathrm{~s}^{-1}$ in the ion diamagnetic drift direction typically measured [13]. Note in AUG the ion diamagnetic drift direction is (poloidally) downward (i.e. grad- $\theta$ is upward) on the low field side for standard $-B_{\mathrm{T}}$ and co-NBI injection $+I_{\mathrm{p}}$ operation - where positive is defined as toroidally counter-clockwise when viewed from above, as shown in figure 1.

Inside the plasma boundary or separatrix the strong pressure gradient associated with the edge pedestal $\left(1<\rho_{\text {pol }} \lesssim 0.85\right)$ provides the drive for electron drift wave (EDW) like turbulence (c.f. [14, 15] and reference therein). The dispersion relation is linear at low $k$ with $v_{\mathrm{ph}}=v_{\mathrm{gr}} \lesssim v_{\mathrm{e}}^{*}=\rho_{\mathrm{s}} c_{\mathrm{s}} / L_{\mathrm{n}}$ in the electron direction $\left(\rho_{\mathrm{s}}\right.$ is the ion Larmor radius at the sound speed $c_{\mathrm{s}}$ and $L_{\mathrm{n}}=(\nabla \ln n)^{-1}$ the density scale length); but can become non-linear for $k_{\perp} \rho_{\mathrm{s}}>0.3$ with even a negative group velocity $v_{\text {gr }}[16,17]$. For example at typical Doppler probed $k_{\perp}>8 \mathrm{~cm}^{-1}$ the phase velocity is small at $v_{\mathrm{ph}} \sim O\left(0.1 v_{\mathrm{e}}^{*}\right)$.

Inside of the density pedestal - the plasma core $\left(\rho_{\text {pol }} \lesssim 0.85\right)$, the ion and electron temperature gradients become the major drive for instabilities leading to ion and electron 


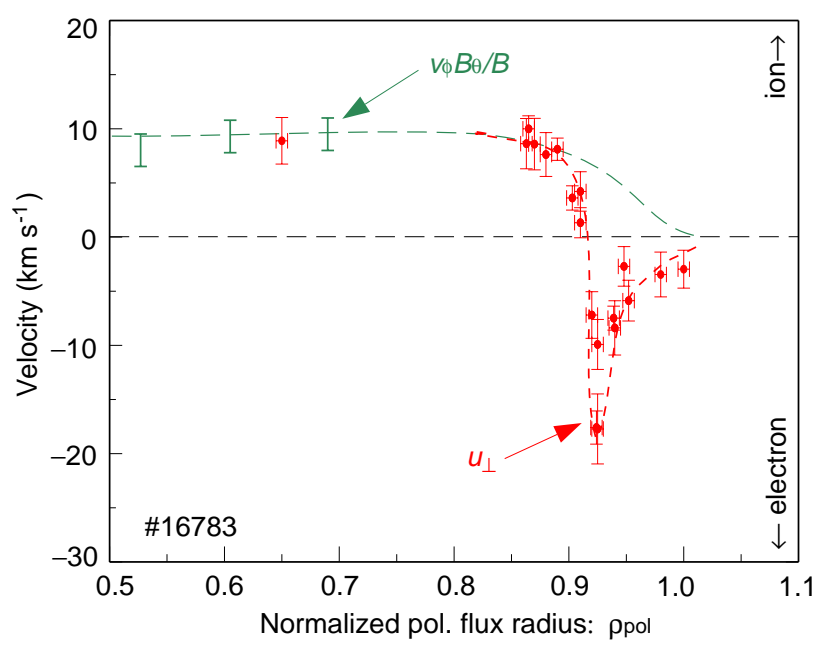

Figure 3. Edge $u_{\perp}$ (red $\bullet$ ) and perpendicularly mapped toroidal (impurity) fluid velocity $v_{\phi} B_{\theta} / B$ (green - - - ) vs normalised radius $\rho_{\text {pol }}$ for NBI H-mode shot \#16783.

temperature gradient (ITG and ETG) turbulence together with turbulence associated with trapped electrons (TEM) (c.f. [14, 18]). ITG and TEM turbulence appear mostly in the long wavelength range $k_{\perp} \rho_{\mathrm{s}} \approx 0.3$ while ETG turbulence is expected at much shorter wavelengths $k_{\perp} \rho_{\mathrm{s}} \approx 10$. There are no simple formulas for the ITG or TEM phase velocities, however, numerical simulations again indicate that ITG turbulence at least has a linear dispersion with a group velocity (in the ion direction) $v_{\mathrm{gr}} \sim O(3) \rho_{\mathrm{s}} c_{\mathrm{s}} / R$ where $R$ is the major radius $[19,20]$. Generally in ohmic and L-mode plasmas $R>L_{\mathrm{n}, \mathrm{T}}$ so that the group velocity may be smaller than the corresponding diamagnetic velocity. Using nonlinear gyrofluid and gyrokinetic simulations Bravenec [21] also finds poloidal phase velocities of $\omega / k_{\theta} \sim 0.3 v_{\mathrm{i}}^{*}$. For TEM turbulence numerical simulations indicate phase or group velocities of the order of a few hundred $\mathrm{ms}^{-1}$ to $1000 \mathrm{~m} \mathrm{~s}^{-1}$ (in the electron direction) depending on the temperature ratio $T_{\mathrm{e}} / T_{\mathrm{i}}[3]$.

A transition region can also be defined between the edge and core which is associated with the pedestal where the strong second radial derivatives in density and temperature together with shearing in the plasma rotation can play additional roles in the turbulence behaviour, for example the formation of zonal flows [22, 23].

Note in the edge the turbulence is electromagnetic in nature while in the core it is believed to be mostly electrostatic. Also the transition from one form of dominant turbulence to another is not well defined and may well be gradual. It is distinctly possible that more than one type of turbulence may exist at any particular place and time depending on the plasma configuration and parameters [24], although most probably not in the same wavenumber range. 


\section{Results}

\section{1. $u_{\perp}$ rotation profiles}

Figure 3 shows a typical low field side radial profile of the rotation velocity $u_{\perp}$ for a $4.5 \mathrm{MW}$ co-NBI H-mode discharge \#16783 $\left(B_{\mathrm{T}}=-2.0 \mathrm{~T}, I_{\mathrm{p}}=+1.0 \mathrm{MA}\right) . u_{\perp}$ is computed from $f_{\mathrm{D}}$ using the vacuum wavelength $\lambda_{\mathrm{o}}$ and the geometric tilt angle from flux surface reconstructions. The mean Doppler shift $f_{\mathrm{D}}$ is obtained by least squares peak fitting to an ensemble averaged complex amplitude $A \exp (i \phi)$ spectrum - with a resulting typical standard error of $\pm 5 \%$. The radial position of the measurement is obtained from the cold plasma approximation for the cutoff using a best fit density profile from Thomson scattering, DCN interferometry, Li-beam and FM profile reflectometry data when available. A beam tracing code (TORBEAM [25]) is used to correct the cutoff position for refraction effects - which can be particularly significant in O-mode for discharges with a steep edge density gradient. The error bars indicated in the figures include the measurement errors, as discussed in [2], but make no allowance for corrections to $k_{\perp}$ and localization due to plasma/wavefront curvature - which recent theory suggest may become significant in the deep core region [26]. Further corrections due to theoretically predicted multiple scattering effects are unlikely to be necessary due to the expected low turbulence level (despite a longer propagation path to the core), plus the absence of strong velocity shearing and the smooth convex density profile in the core region [27].

In the SOL $u_{\perp}$ is typically $1-2 \mathrm{~km} \mathrm{~s}^{-1}$ and always flows in the ion direction, i.e. a positive or outward pointing radial electric field. Across the separatrix $u_{\perp}$ reverses to the electron direction consistent with the familiar negative $E_{\mathrm{r}}$ well $[28,29]$ associated with the steep pedestal pressure gradient in the H-mode edge (i.e. large dominant diamagnetic and poloidal fluid velocities). Typical peak well $u_{\perp}$ values are between $20-60 \mathrm{~km} \mathrm{~s}^{-1}$ depending on the strength of the edge transport barrier. In the core region the momentum driven toroidal rotation from the tangential neutral beam injection becomes the dominant factor in the radial force balance equation

$$
v_{E \times B}=v_{\perp s}-v_{s}^{*}=v_{\phi s} B_{\theta} / B-v_{\theta s} B_{\phi} / B-\nabla P_{s} / q n_{s} B
$$

where $s$ is the (electron, ion or impurity) species. With co-injection $u_{\perp}$ reverses to the ion direction and tends to follow the perpendicular component of the toroidal fluid velocity $\left(C^{6+}\right.$ impurity from CXRS mapped into the perpendicular plane $\left.v_{\phi} B_{\theta} / B\right)$ - as shown by the dashed-line in figure 3. Previously it was shown that reversing either the toroidal $B_{\phi}$ or poloidal $B_{\theta}$ magnetic field resulted in reversals in $u_{\perp}$ consistent with the radial force balance equation [2].

With NBI heating $u_{\perp}$ is typically $10-20 \mathrm{~km} \mathrm{~s}^{-1}$ in the core. However, in the absence of a dominant toroidal rotation, for example discharges with balanced NBI or with ion or electron cyclotron heating (ICRH or ECRH) or even ohmic plasmas, both the core and edge $u_{\perp}$ drop to only a few $\mathrm{km} \mathrm{s}^{-1}$, which is now comparable to expected turbulence phase and poloidal fluid velocities. Figure 4 shows a series of $u_{\perp}$ radial profiles for ohmic 


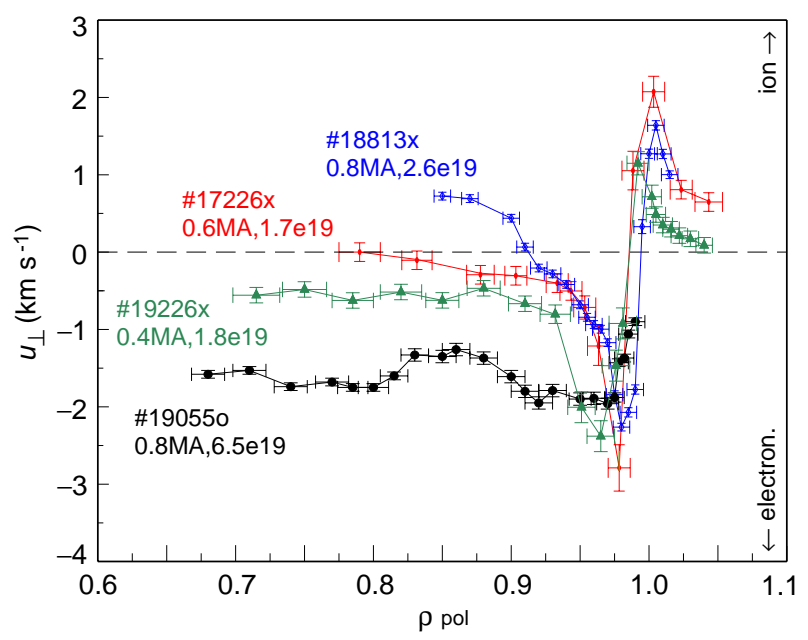

Figure 4. $u_{\perp}$ vs normalised poloidal flux radius $\rho_{\text {pol }}$ for various lower single null ohmic discharges.

shots with various electron density and plasma currents. All discharges are lower single null diverted deuterium plasmas with $-B_{\mathrm{T}}$ and $+I_{\mathrm{p}}$. The profile displays the usual positive and negative peak structure associated with the separatrix and pedestal $\nabla P$ shear regions - which remains remarkably robust for all shots - but the core rotation (i.e. inside the $n_{\mathrm{e}}$ pedestal) can either stay in the electron drift direction at high density or reverse to the ion direction at very low densities. Inside $\rho_{\text {pol }} \approx 0.85$ the profile is notably flat.

\subsection{Collisionality dependence}

The robustness of the ohmic edge profile may possibly be ascribed to the high collisionality of the AUG edge plasmas - for $\rho_{\text {pol }}>0.9$ the collisionality is nearly always above the banana limit - which affects the predominantly drift-wave turbulence behaviour only marginally. The core, on the other hand, can experience much wider variations in collisionality, with consequent stronger effects on the turbulence.

Figure 5 shows the $u_{\perp}$ velocity at $\rho_{\text {pol }} \approx 0.7$ for a range of lower single null ohmic discharges plotted against both the local effective collisionality $\nu_{\text {eff }}$ and the local normalised collisionality $\nu^{*}$. The effective collisionality is defined as

$$
\nu_{\mathrm{eff}}=\nu_{\mathrm{ei}} / \omega_{\mathrm{De}} \approx 10^{-14} R Z_{\mathrm{eff}} n_{\mathrm{e}} T_{\mathrm{e}}^{-2},
$$

where $\omega_{\mathrm{De}}=2 k_{\theta} \rho_{\mathrm{s}} c_{\mathrm{s}} / R$ is the curvature drift frequency (a rough estimate of the ITG growth rate) and $R=1.68 \mathrm{~m}$ and a constant effective charge of $Z_{\text {eff }}=2$. The normalized collisionality on the other hand is defined here as

$$
\nu^{*}=\nu_{\mathrm{ei}} /\left(\epsilon \omega_{\mathrm{be}}\right)=6.92 \times 10^{-18} q R Z_{\mathrm{eff}} n_{\mathrm{e}} \ln \Lambda T_{\mathrm{e}}^{-2} \epsilon^{-3 / 2}
$$

where the local inverse aspect ratio $\epsilon=r / R=0.166$ at $\rho_{\text {pol }}=0.7$ and $\ln \Lambda \approx 17$. The density ranges between $1.25-7.4 \times 10^{19} \mathrm{~m}^{-3}$ with a corresponding $T_{\mathrm{e}}$ between $1070-350 \mathrm{eV}$. $B_{\mathrm{T}}$ is roughly constant between $2.0-2.3 \mathrm{~T}$ but $I_{\mathrm{p}}$ varies between 

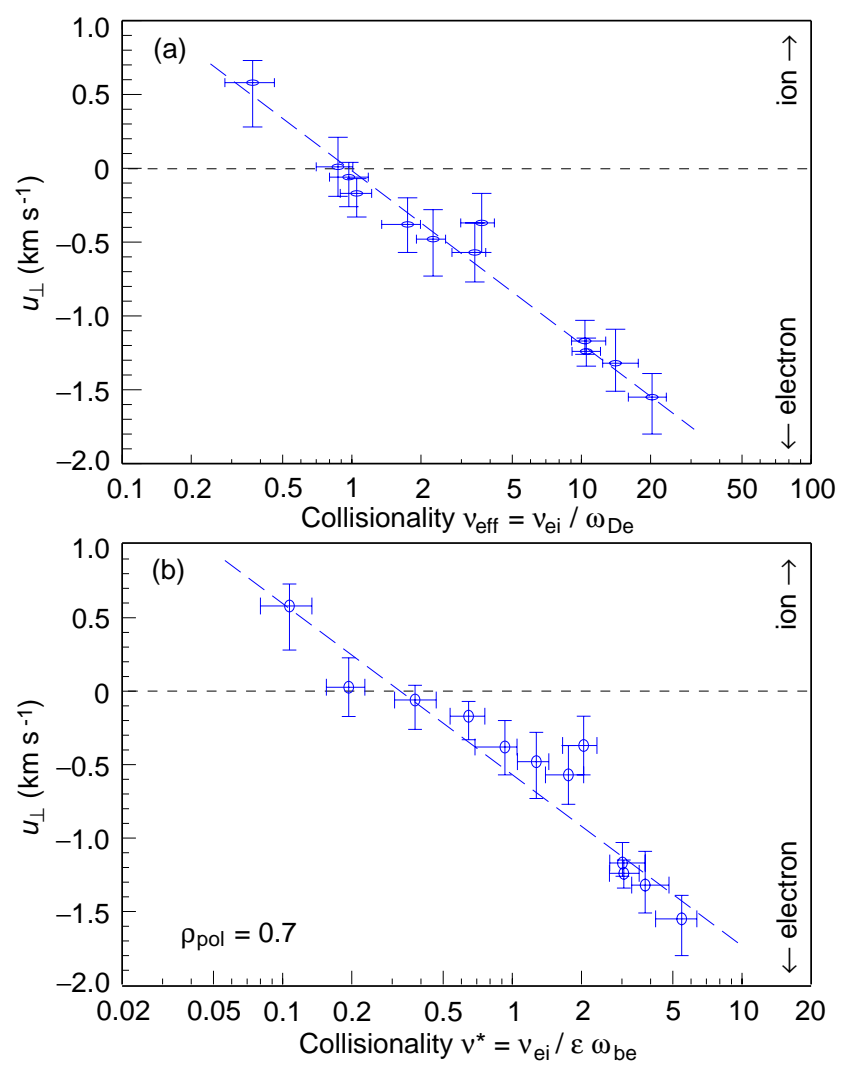

Figure 5. $u_{\perp}$ at $\rho_{\mathrm{pol}} \approx 0.7$ for various ohmic discharges with varying $n_{\mathrm{e}}, T_{\mathrm{e}}$ and $I_{\mathrm{p}}$ vs (a) effective collisionality $\nu_{\text {eff }}$ and (b) normalized collisionality $\nu^{*}$.

$0.4-0.8 \mathrm{MA}$ giving a significant variation in local safety factor $q$. The result is a collisionality scan covering almost two orders of magnitude. The remarkable feature is the practically linear variation of $u_{\perp}$ against $\log (\nu)$, that is an exponential dependence, rather than an inverse dependence that many parameters display with collisionality. The direction of dependence is such that at low collisionality $u_{\perp}$ is in the ion drift direction but reverses smoothly to the electron drift direction with increasing collisionality. Note that the points line up better against $\nu_{\text {eff }}$ rather than $\nu^{*}$, which suggests the rotation velocity $u_{\perp}$ may depend more on the physics of the turbulence (i.e. the growth rate factor $\omega_{\text {De }}$ ) than on neoclassical transport (i.e. the bounce frequency $\omega_{\mathrm{be}}$ ).

\subsection{Turbulence phase velocity}

Numerical simulations of these plasmas using gyrofluid and gyrokinetic codes predict that TEM turbulence should become linearly stable at high densities/collisionalities while ITG turbulence remains unstable $[3,30]$. There are also experimental observations, e.g. on density peaking and heat flux, which appear to confirm this transition hypothesis $[3,4]$. A transition in the dominant turbulence with increasing collisionality would appear as a reversal in the $v_{\mathrm{ph}}$ direction from the electron to the ion drift direction. This is shown in figure 6 with results from the linear gyrokinetic code Gs2 [31]. The 


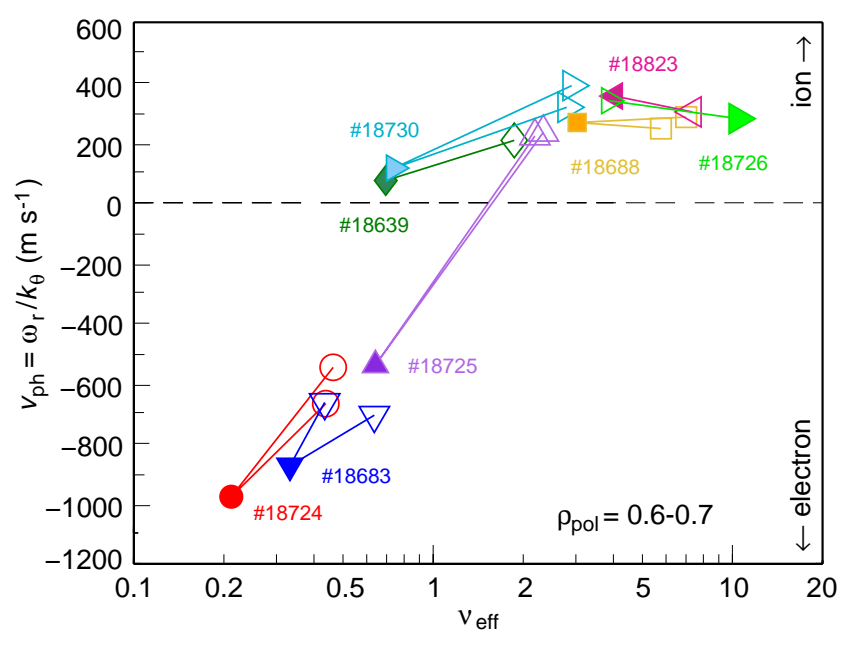

Figure 6. Computed turbulence phase velocity $v_{\text {ph }}$ from GS2 code vs effective collisionality $\nu_{\text {eff }}$ for $\rho_{\text {pol }}=0.6-0.7$. Open symbols $=$ ohmic, solid $=$ ECRH.

phase velocity is computed from the real part of the dominant instability frequency corresponding to the poloidal wavenumber at which the growth rate $\gamma / k_{\perp}^{2}$ is maximum $\left(v_{\mathrm{ph}}=\omega_{r} / k_{\theta}\right)$ plotted vs the effective collisionality $\nu_{\text {eff }}$ for $\rho_{\text {pol }}=0.6-0.7$. The definition of $\nu_{\text {eff }}$ is such that in these experimental conditions a value of 1 roughly corresponds to the boundary between TEM and ITG.

Each point in figure 6 is a simulation of an individual experimental discharge with temperature, density and $q$ profiles taken from measured data. The discharges were part of a programme of controlled experiments to investigate density peaking, particle thermo-diffusion and electron heat diffusivities in ohmic and L-mode conditions [3]. The open symbols are from ohmic phases of the discharges while the solid symbols are from ECRH phases. These are discussed more fully in the section on perturbation experiments. In these simulations the effective charge $Z_{\text {eff }}$ is modelled as a smooth decreasing function of density from 2.5 at low density to 1.5 at high density.

Plotting the measured $u_{\perp}$ (black open circles) and computed $v_{\mathrm{ph}}$ (red full circles) velocities together against $\nu^{*}$ in figure 7 shows the phase velocity transition from TEM to ITG dominance occurs at a similar $\nu^{*}$ value as the $u_{\perp}$ transition - but in opposite directions! Subtracting the computed phase velocity from the measured rotation (assuming a linear fit) gives an estimate of the corresponding $v_{E \times B}=u_{\perp}-v_{\mathrm{ph}}$ (shown as a green line). Note that the $u_{\perp}$ is not a flux surface averaged value but a local measurement on the poloidal low field side, slightly above the mid-plane. The roughly constant $v_{\mathrm{ph}}$ in the ITG regime means $v_{E \times B}$ follows the $u_{\perp}$ until the transition to TEM where the phase velocity reversal creates a corresponding jump in $v_{E \times B}$. Since $B_{\mathrm{T}}$ is negative and approximately constant for the data shown this implies that the radial electric field decreases with collisionality and actually reverses from negative to positive with the transition from ITG to TEM turbulence. It is a strong statement to say that the turbulence is dictating the $E \times B$ velocity but there appears to be a clear inference. The next question of relevance is what is the behaviour and role of the background bulk 


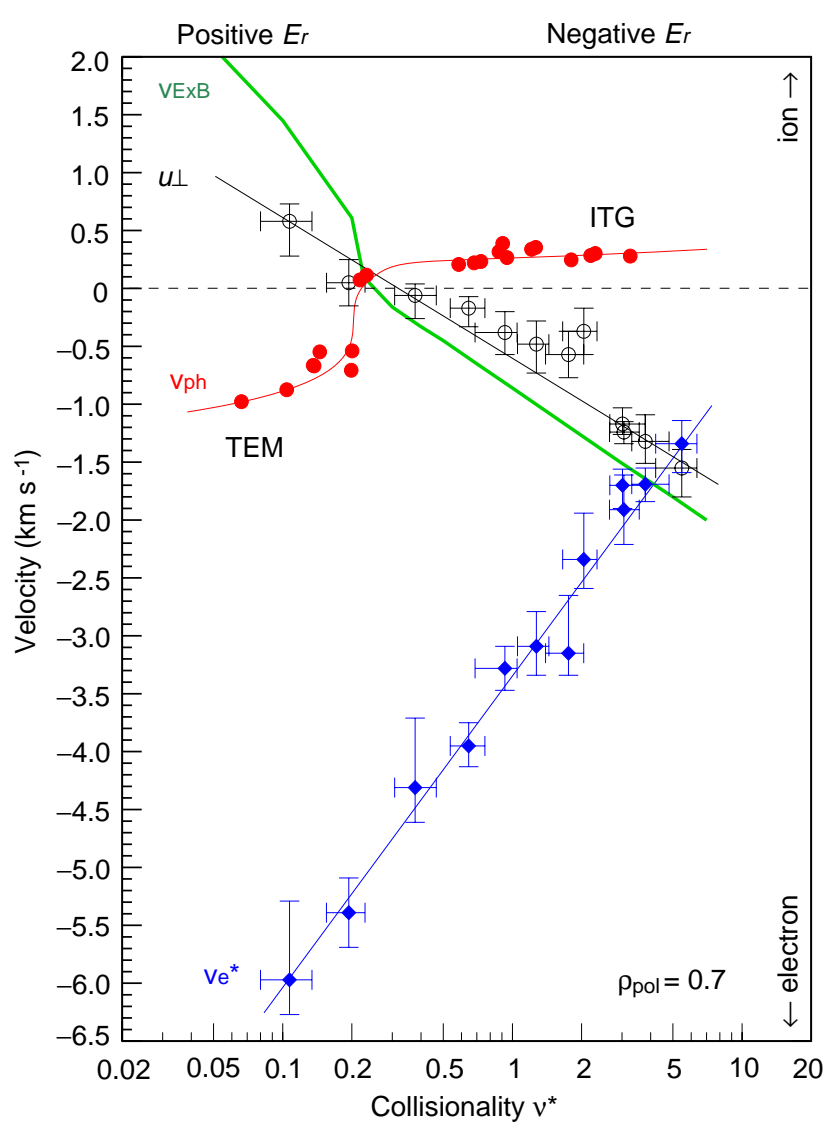

Figure 7. Core $u_{\perp}($ black $\bigcirc)$, computed phase $v_{\text {ph }}\left(\right.$ red $\bullet$ ), extracted $v_{E \times B}$ (green - ) and measured electron diamagnetic $v_{\mathrm{e}}^{*}$ (blue $\checkmark$ ) velocities vs normalized collisionality $\nu^{*}$ at $\rho_{\text {pol }} \approx 0.7$.

plasma fluid rotation.

Also plotted in figure 7 is the electron diamagnetic velocity $v_{\mathrm{e}}^{*}=-\nabla P_{\mathrm{e}} /\left(n_{\mathrm{e}} e B\right)$ (blue diamonds) computed using $T_{\mathrm{e}}$ from ECE and $n_{\mathrm{e}}$ from best fit to TS and DCN data. $v_{\mathrm{e}}^{*}$ varies from $-1.4 \mathrm{~km} \mathrm{~s}^{-1}$ at high $\nu^{*}$ to $-6 \mathrm{~km} \mathrm{~s}^{-1}$ at low $\nu^{*}$. From the radial force balance equation $v_{E \times B}=v_{\perp s}-v_{s}^{*}$ one can deduce the perpendicular fluid velocity

$$
v_{\perp s}=v_{\phi s} B_{\theta} / B-v_{\theta s} B_{\phi} / B .
$$

At low collisionality $v_{\perp \mathrm{e}} \approx-7.5 \mathrm{~km} \mathrm{~s}^{-1}$ rising to $v_{\perp \mathrm{e}} \approx 0.6 \mathrm{~km} \mathrm{~s}^{-1}$ at the highest collisionality. Scaling $\nabla T_{\mathrm{i}}$ from the measured $\nabla T_{\mathrm{e}}$ gives corresponding $v_{\perp \mathrm{i}}$ values of $\approx 4.8 \mathrm{~km} \mathrm{~s}^{-1}$ (ion direction) at low $\nu^{*}$ to $-0.7 \mathrm{~km} \mathrm{~s}^{-1}$ (electron) at high $\nu^{*}$. If the toroidal fluid velocity $v_{\phi \mathrm{i}}$ is small, and thus practically negligible when multiplied by $B_{\theta} / B \sim O(0.16)$ at $\rho_{\text {pol }}=0.7$ compared to the poloidal fluid with $B_{\phi} / B \sim O(0.98)$, then consequently poloidal ion fluid velocities of $v_{\theta \mathrm{i}} \approx-v_{\perp \mathrm{i}}$ would be required.

\subsection{Poloidal fluid velocity}

Unfortunately experimental measurements of the poloidal fluid velocity are not available in ohmic discharges in AUG. Although measurements using CXRS on heating beams 

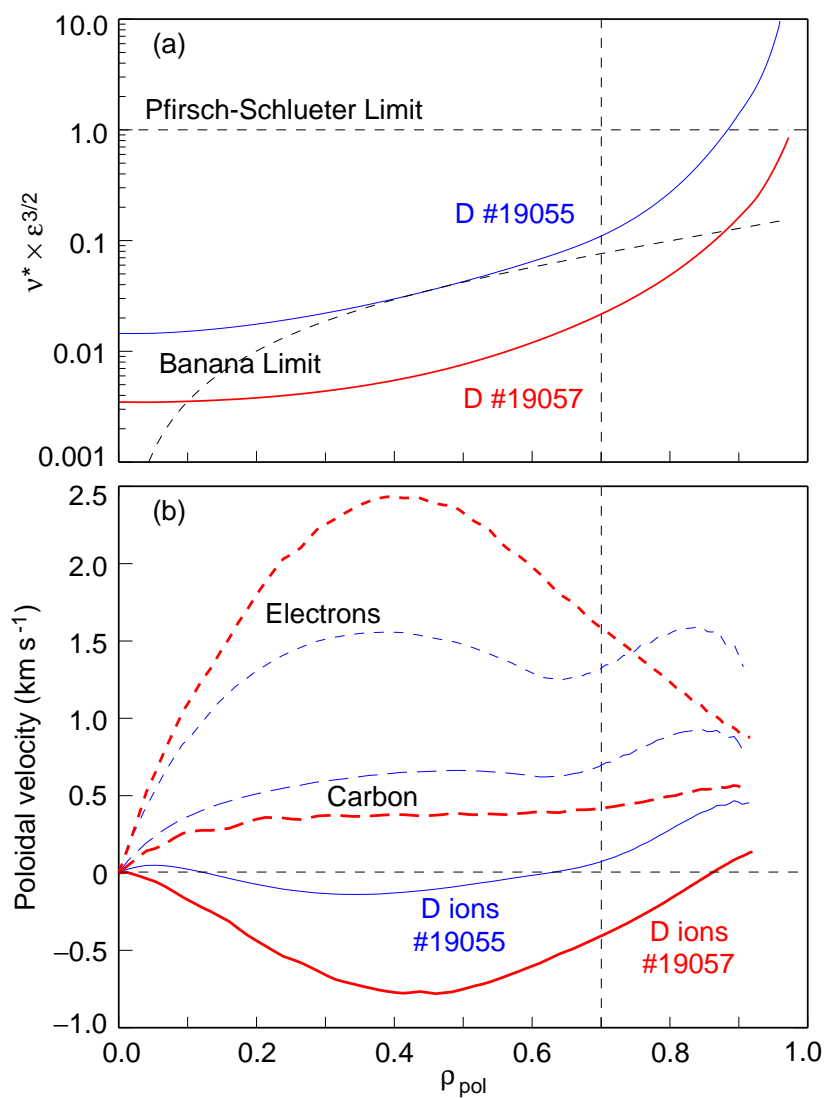

Figure 8. (a) Collisionality $\nu^{*} \epsilon^{3 / 2}$ and (b) poloidal fluid velocity profiles, D ion (-), C ion (-- ), electron (- - -) for low density \#19057 (red/dark) and high density \#19055 (blue/light) ohmic shots from NEOART code using scaled $T_{\mathrm{i}}$ and $Z_{\text {eff }}$.

in L-mode discharges gives values of $v_{\theta} \approx-2 \pm 6 \mathrm{~km} \mathrm{~s}^{-1}$ [32]. A few kilometres per second are also typical values reported from DIII-D [28, 33] in L-mode, JFT-2M [34], Heliotron-E [35], CHS stellarator [36] with NBI and ECRH L-mode conditions. These $v_{\theta}$ values would be of the right order of magnitude required for the ohmic discharges.

However, to assess the collisionality dependence neoclassical calculations have been performed for the conditions shown in figure 7 using two codes, NEOART and NCLASS. The NEOART code by A.G.Peeters calculates the collisional transport coefficients for an arbitrary number of impurities, including inter-species collisions. It solves a set of linear coupled equations for "reduced velocities" (described in [37]) for all collisional regimes. The classical fluxes are those given by Hirshman and Sigmar [38], as is the Pfirsch-Schlüter contribution, with the banana plateau contribution given by Houlberg [39]. Details of the NCLASS code can be found in the literature [41, 39]. Both codes use experimental $T_{\mathrm{e}}$ and $n_{\mathrm{e}}$ profile data as input (with NCLASS using an ASTRA [42] computed equilibrium). In the absence of measurements, $T_{\mathrm{i}}$ and impurity profiles are modelled using experimentally consistent values.

Figure 8 shows the radial profiles of the computed collisionality $\nu^{*} \epsilon^{3 / 2}$ and poloidal fluid velocity using the NEOART code for the two extreme collisionality cases shown in 


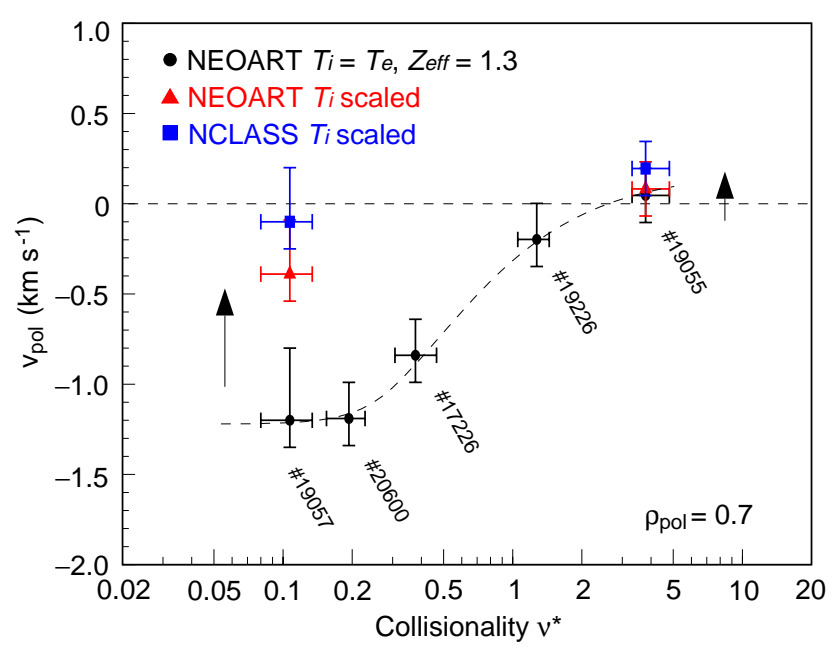

Figure 9. Neoclassical poloidal fluid velocity vs collisionality $\nu^{*}$ from NEOART with $T_{\mathrm{i}}=T_{\mathrm{e}}$ and $Z_{\mathrm{eff}}=1.3\left(\right.$ black $\bullet$ ), NEOART with $T_{\mathrm{i}}$ and $Z_{\text {eff }}$ scaled (red $\left.\boldsymbol{\Delta}\right)$ and NCLASS with $T_{\mathrm{i}}$ and $Z_{\text {eff }}$ scaled (blue

figure 7 - with the assumption of zero toroidal rotation, $v_{\phi}=0$. For the low density ohmic simulation (\#19057 - red) the ion temperature profile was scaled from the electron with $T_{\mathrm{i}}=0.55 T_{\mathrm{e}}$ and $Z_{\mathrm{eff}}=2.5$ while for the high density run (\#19055- blue) $T_{\mathrm{i}}=0.85 T_{\mathrm{e}}$ and $Z_{\text {eff }}=1.3$. These scalings are in line with previous measured $T_{\mathrm{i}}$ profiles from similar shots [3].

The poloidal rotation generally peaks around mid-radius. Carbon ions and electrons rotate together while the Deuterium ions rotate in the opposite direction. Increasing the collisionality sees both the $D$ and $C$ ion velocities becoming more positive while the electron velocity decreases. In the plasma edge the $D$ ion velocity actually reverses sign and all species flow in the same direction (c.f. [40]). The overall velocities are in the range of $0.5-2 \mathrm{~km} \mathrm{~s}^{-1}$.

These two discharges were also simulated using the NCLASS code with identical input profiles as for NEOART. The comparative results are shown in figure 9 where the $D$ ion poloidal fluid velocity at $\rho_{\text {pol }}=0.7$ is plotted vs collisionality $\nu^{*}$. The NEOART values from figure 8 are shown as red triangles while the blue squares are the NCLASS results. Both codes give similar values for the $D$ ions, although the NCLASS values are generally more positive at both collisionality ends. Also shown are the results of a full collisionality scan using the NEOART code (black circles) for selected shots from figure 7 . To simplify the full $\nu^{*}$ scan the ion temperature is set equal to the electron $T_{\mathrm{i}}=T_{\mathrm{e}}$ and the effective charge to $Z_{\mathrm{eff}}=1.3$. The $D$ ion $v_{\theta}$ scales monotonically with $\nu^{*}$, saturating at low $\nu^{*}$. Comparing with the scaled $T_{\mathrm{i}}$ results shows that decreasing the ion temperature makes the poloidal rotation more positive and increasing $Z_{\text {eff }}$ makes $v_{\theta}$ smaller. Although the overall variation with collisionality is consistent with the required $v_{\perp \mathrm{i}}$, the neoclassical values are almost an order of magnitude too small, particularly at the low collisionality end. Similar disagreements between neoclassical predictions and experimental observations have also been reported for DIII-D [41] and JET [43]. 


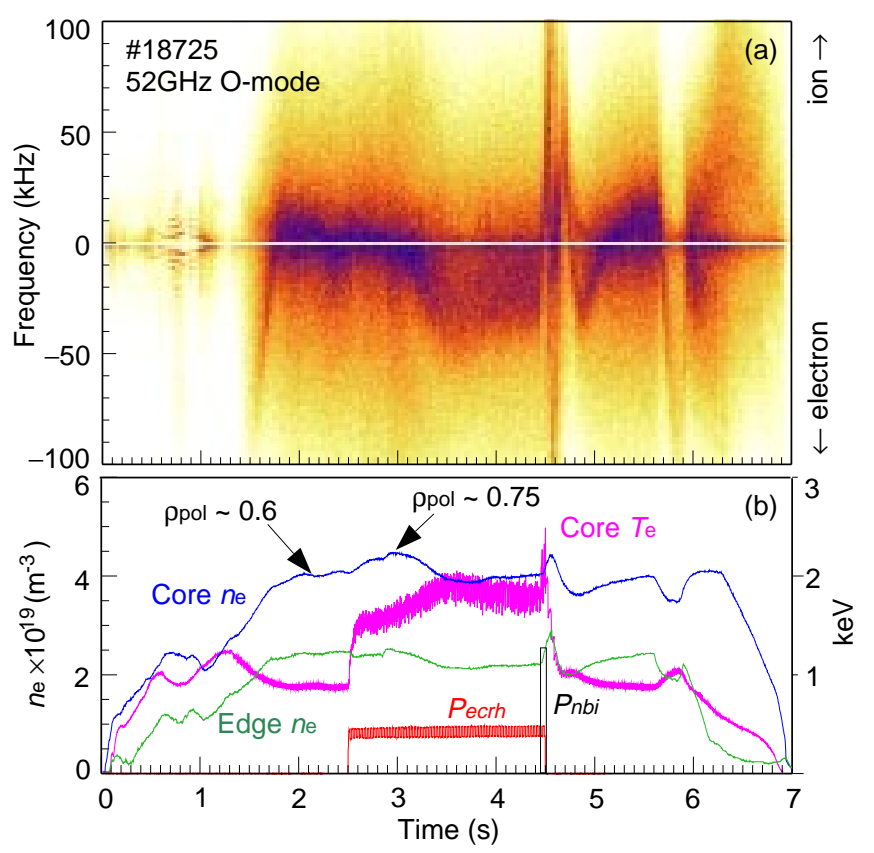

Figure 10. (a) Spectrogram of complex amplitude signal from FLV reflectometer, and (b) time traces of core and edge line average density, core electron temperature and heating waveforms for shot \#18725.

While it is encouraging that the neoclassical predictions show the necessary trend of $v_{\theta}$ with collisionality consistent with experiment, the assumption of zero toroidal rotation needs to be reconsidered - since in the experiment the perpendicular fluid and diamagnetic velocities must balance the $E \times B$ velocity. The neoclassical poloidal simulations also fail to throw further light on the interaction between the turbulence and the background plasma rotation. We address this question in a second set of experiments where perturbations in the collisionality are induced by the application of small amounts of additional ECRH electron heating power.

\section{ECRH perturbation experiments}

Since $\nu^{*} \propto n_{\mathrm{e}} / T_{\mathrm{e}}^{2}$ there are two possible experimental approaches to induce a turbulence transition: a density ramp using gas puffing for example, or a temperature perturbation using central ECRH. A density variation produces a linear change in $\nu^{*}$ and a corresponding variation in the Doppler shift $f_{\mathrm{D}}$ as shown in figure 5. A temperature variation however appears to be more subtle and its effect is found to depend on the density and its proximity to the turbulence transition, $\nu_{\text {eff }} \sim 1$ in figure 6 .

For example, figure 10 shows a frequency vs time spectrogram of complex amplitude, $A \exp (i \phi)$, fluctuations from the O-mode FLV reflectometer channel at $52 \mathrm{GHz}$ during an $I_{\mathrm{p}}=0.8 \mathrm{MA}$ and a (medium) line averaged density of $\sim 4 \times 10^{19} \mathrm{~m}^{-3}$ (\#18725). This density is close to the critical transition where TEM and ITG turbulence are expected to co-exist. Below the spectrogram are time traces of the line average density from 

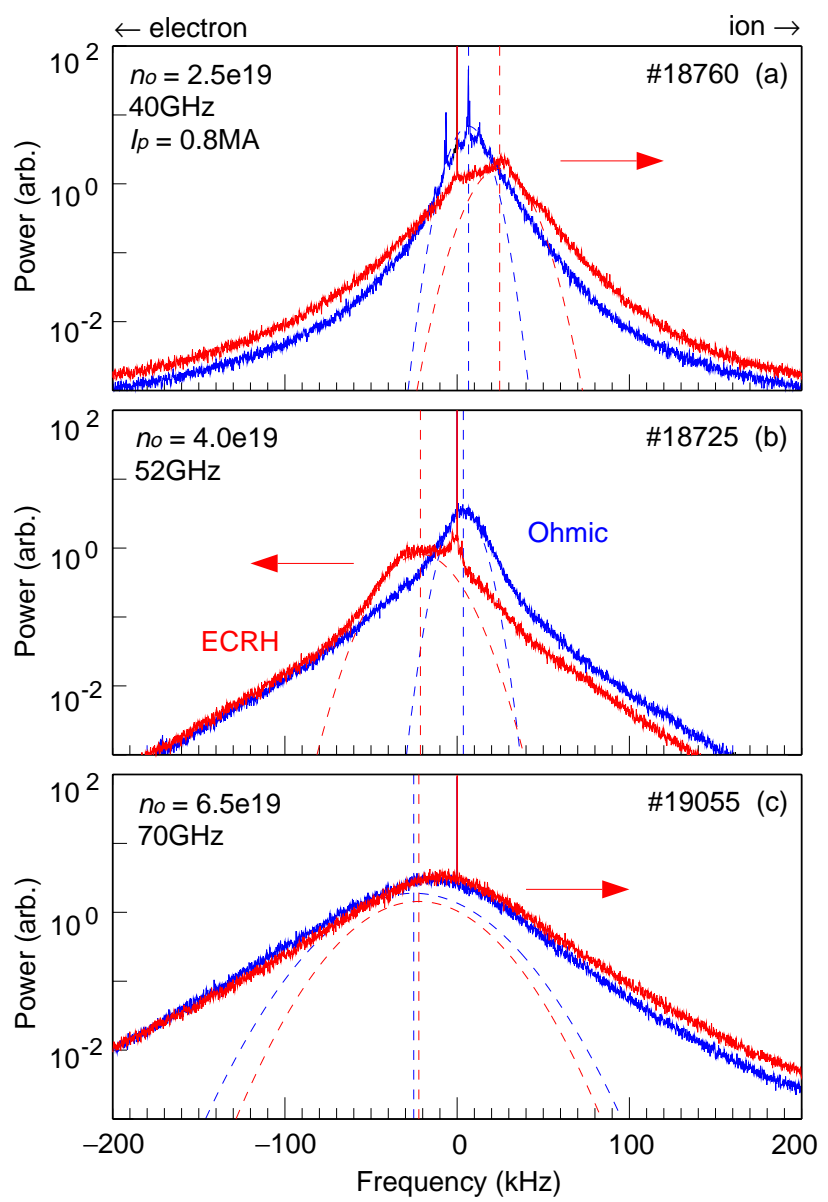

Figure 11. Doppler spectra for ohmic (blue) and ECRH (red) phases at (a) low, (b) medium and (c) high line average densities, $\rho_{\mathrm{pol}}=0.6-0.7$. The dashed curves are fitted Gaussians to the spectral peaks.

core and edge interferometer channels, plus core $T_{\mathrm{e}}$ from ECE. Between 2.5 and 4.5 seconds 0.8 MW of on-axis ECRH power is applied. During the initial ohmic phase the spectrogram shows the core $u_{\perp}$ is in the ion direction but reverses to the electron direction with the application of ECRH. The ECRH raises the core $T_{\mathrm{e}}$ (and its gradient) which lowers $\nu_{\text {eff }}$ by a factor of 2 or so (c.f. the open to closed symbols in figure 6 ). The movement in the electron direction is therefore inconsistent with the $u_{\perp}$ dependence in figure 5 , but is consistent with the $v_{\mathrm{ph}}$ dependence in figure 6 . Note the gradual $f_{\mathrm{D}}$ change at the start of the ECRH phase is the result of the density rise and fall which moves the reflectometer cutoff layer out from $\rho_{\text {pol }} \approx 0.6$ to 0.75 and back. Similar discharges display more abrupt changes. The rapid change in $f_{\mathrm{D}}$ at the end of the ECRH phase is due to a short 2.5 MW NBI beam-blip.

On the other hand, figure 11 shows reflectometer spectra at $40 \mathrm{GHz}$ (FLQ), $52 \mathrm{GHz}$ (FLV) and $70 \mathrm{GHz}(\mathrm{FLV})$ for three line average densities, 2.5, 4.0 and $6.5 \times 10^{19} \mathrm{~m}^{-3}$ respectively, with the same plasma current $I_{\mathrm{p}}=0.8 \mathrm{MA}$ and $B_{\mathrm{T}}=-2.35 \mathrm{~T}$. The cutoff layers are at similar $\rho_{\text {pol }}=0.6-0.7$ in each case. The blue spectra are during the ohmic phase of the discharges while the red spectra are during the ECRH phase. 
In the ohmic phase, with increasing density the Doppler shift moves from the ion to the electron direction - consistent with increasing collisionality as indicated in figure 5 . At low density, well below the critical value for turbulence transition (i.e. TEM and ITG turbulence present but TEM has the dominant growth rate) the $f_{\mathrm{D}}$ shift with ECRH moves in the ion direction. This suggests that the change in fluid velocity $\Delta v_{\perp \mathrm{i}}$ associated with neoclassical effects dominate over any $\Delta v_{\text {ph }}$ change associated with the turbulence. In figure 6 there is only a small $\Delta v_{\text {ph }}$ at low $\nu_{\text {eff }}$ - as shown by the lines linking the open to closed symbols. At high density, that is well above the turbulence transition (i.e. ITG turbulence only) the $f_{\mathrm{D}}$ also moves very slightly in the ion direction with the ECRH. This again is consistent with the roughly constant $v_{\mathrm{ph}}$ at high $\nu_{\text {eff }}$ indicating $\Delta v_{\mathrm{ph}} \ll \Delta v_{\perp \mathrm{i}}$. Note also in this case the excessive broadening in turbulence frequency spectra - beyond that due to rotational broadening alone - which implies a change in the underlying turbulence $k$-spectrum and hence a change in turbulence.

However, at medium density (i.e. close to the transition with TEM and ITG turbulence present) the Doppler shift $f_{\mathrm{D}}$ moves in the electron direction with the ECRH (as shown in the spectrogram of figure 10). This is the apparently anomalous case. It could be explained with a $\Delta v_{\mathrm{ph}} \gg \Delta v_{\perp \mathrm{i}}$. Certainly figure 6 indicates a large step in $v_{\text {ph }}$ across the transition region, but in the ohmic case this is balanced by an equal and opposite step in the $v_{E \times B}$. The ECRH phase therefore appears to move away from the ohmic $u_{\perp}$ line shown in figure 7 . This might be due to the steepening of the electron temperature gradient during the ECRH which may drive the TEM turbulence harder resulting in a larger $\Delta v_{\mathrm{ph}}$ and hence a stronger effect in the $u_{\perp}$ behaviour. In any case, the medium density spectra with and without ECRH show the clearest experimental evidence of a transition from ITG to TEM dominated turbulence.

\section{Discussion and conclusions}

The GS2 numerical simulations predict turbulence phase velocities of the order of $200-1000 \mathrm{~m} \mathrm{~s}^{-1}$, which are of the same order of magnitude as $u_{\perp}$ in all but high powered neutral beam driven shots. Therefore it will certainly be necessary to correct the core Doppler reflectometer $u_{\perp}$ values before extracting the $E_{\mathrm{r}}$. Even in strong NBI heated discharges the core $u_{\perp}$ is only $10-20 \mathrm{~km} \mathrm{~s}^{-1}$ so that ignoring a phase velocity of $1-2 \mathrm{~km} \mathrm{~s}^{-1}$ would generate a $5-10 \%$ error in $v_{E \times B}$. In the current absence of $v_{\mathrm{ph}}$ values in $\mathrm{H}$-mode conditions, this may have to serve as an acceptable error bar. Fortunately the plasma edge region appears to require less attention since the prevailing higher collisionality and predominance of electron drift-wave turbulence - together with sufficiently large tilt angles, i.e. large probed $k_{\perp}$ - practically guarantee negligible phase velocities.

The wider variability of core collisionality, however, makes for interesting Doppler reflectometer measurements. The core certainly offers the only possibility of separating the phase velocity from the bulk plasma rotation, particularly in low rotation conditions such as ohmic or ECRH L-mode. By varying the plasma current and density on a shot- 


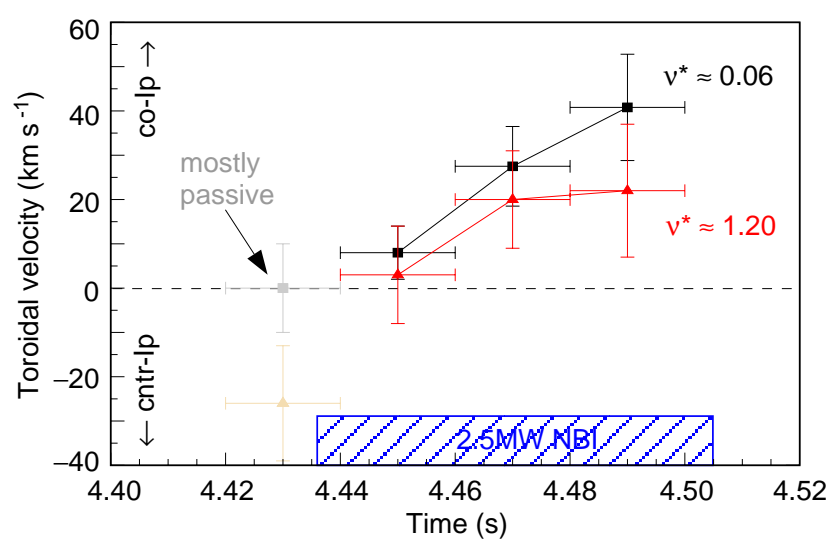

Figure 12. Carbon impurity toroidal rotation velocity from active CXRS as a function of time during a $69 \mathrm{~ms}$ long, $2.5 \mathrm{MW}$ NBI beam blip at end of ECRH at $\rho_{\text {pol }}=0.7$ in low (\#18724) and high (\#18726) collisionality discharges. Time bar indicates integration window.

by-shot basis a collisionality scan over almost two orders of magnitude could be obtained in AUG ohmic conditions, covering the expected region for a transition between ITG and TEM dominated core turbulence. To experimentally observe a transition directly in a key turbulence parameter would provide a strong validation of prevailing turbulence theories and numerical simulation codes. Thus, the measured decline and reversal in the $u_{\perp}$ velocity direction coinciding with the theoretically predicted phase velocity reversal provides a good indication of the validity of current turbulence modelling. Similar, although more restricted, $u_{\perp}$ results were also obtained with ohmic density ramps in individual shots, but it is generally not possible to get the same range of collisionality variation and cutoff layer coverage in the core during a single discharge.

Subtracting the computed $v_{\mathrm{ph}}$ from the measured $u_{\perp}$ gives a $v_{E \times B}$ - and hence $E_{\mathrm{r}}$ with a large jump across the turbulence transition. Since the diamagnetic velocity shows no corresponding jump the $v_{E \times B}$ jump must be matched by a corresponding change in the bulk perpendicular fluid velocity. If the simulation phase velocities are correct this would infer that the turbulence is strongly interlinked, not just with $E_{\mathrm{r}}$, but also with the bulk rotation of the plasma, meaning, the turbulence is not just set or dictated by the background but also interacts and affects it in a close relationship.

The measured diamagnetic velocity varies greatly with collisionality, being less than the $E \times B$ velocity at high collisionality, but factors of three larger at low collisionality. To balance the smaller $E \times B$ velocity the bulk perpendicular fluid velocity must make equally large variations, even reversing direction at high collisionality. Neoclassical predictions of the $D^{+}$poloidal fluid velocity give the required behavioural trend, complete with direction changes at the correct collisionality, but are factor of ten too small, particularly at low collisionality. It is possible that anomalous poloidal rotation might be driven by the turbulence itself, for example by Reynolds stress [45], but it is not clear if such large rotations could result.

In the neoclassical calculations it was assumed that the toroidal rotation was zero, 
however, there is evidence from Alcator C-Mod, for example, that toroidal rotation can be significant even in ohmic discharges [44]. The effect of a toroidal rotation will be most significant at low collisionality, but it would need to be substantial - of the order of $30-47 \mathrm{~km} \mathrm{~s}^{-1}$ at $\rho_{\text {pol }}=0.7$ in the co-direction (with $I_{\mathrm{p}}$ ) at the lowest collisionality - to account for the discrepancy between the predicted perpendicular fluid rotation and the experimental $u_{\perp}$ measurements. Such large toroidal velocities are normally observed in NBI driven L-mode and H-modes (typically low collisionality) but are not expected for ohmic conditions. CXRS toroidal rotation measurements are available for several of the discharges shown in figure 6 using single NBI beam blips (2.5 MW for $69 \mathrm{~ms}$ ) at the end of the ECRH phase. The beam blips are highly perturbative, as indicated in the Doppler spectrum of figure 10, and there is a clear spin-up in the $C^{6+}$ impurity toroidal rotation $v_{\phi}$ from a couple of $\mathrm{km} \mathrm{s}^{-1}$ to around $40 \mathrm{~km} \mathrm{~s}^{-1}$ during the beam blip at $\rho_{\text {pol }} \sim 0.7$ as shown in figure 12. Although the $\pm 10 \mathrm{~km} \mathrm{~s}^{-1}$ error bars are substantial, a definite rise in $v_{\phi}$ is observed with decreasing collisionality. For example, the first real CXRS measurement just after the beginning of the blip - which should be indicative of the maximum ohmic rotation - increases from around $2 \mathrm{~km} \mathrm{~s}^{-1}\left(\nu^{*} \sim 1.2\right)$ to around $7 \mathrm{~km} \mathrm{~s}^{-1}\left(\nu^{*} \sim 0.06\right)$. Nevertheless, the $D^{+}$toroidal rotation would still need to be a factor of 4 to 5 higher to match the required perpendicular rotation. Counter rotation in the ohmic phase also can not be discounted.

The strongest effects observed in the Doppler reflectometer spectra were for conditions where TEM and ITG are predicted to co-exist. Co-existence suggests that two Doppler peaks might be observable in the reflectometer spectra, corresponding to

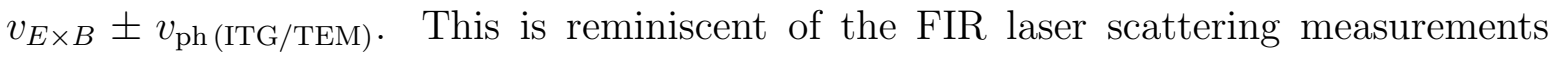
on TEXT which showed ion and electron peaks during the transition from linear to saturated ohmic confinement conditions [46]. However, Doppler reflectometry has the additional advantage of radial localization with high $k_{\perp}$. Initial attempts with optimal conditions for TEM/ITG co-existence and ECRH modulation to switch on/off the TEM have not yet revealed convincing double Doppler peaks, or a modulation of $u_{\perp}$ about a mean $v_{E \times B}$. This may possibly be due to too small a phase velocity difference $v_{\mathrm{ph}(\mathrm{ITG})}-v_{\mathrm{ph} \text { (TEM) }}$, or an insufficient diagnostic spectral resolution. It might also indicate peaks of incomparable amplitude - that is to say that the dominant form of turbulence strongly suppresses the weaker one. This is a topic of further study.

\section{Acknowledgments}

We thank W.Suttrop and E.Schmid for assistance with reflectometer hardware, W.Dorland and M.Kotschenreuther for provision of the GS2 code, C.Maggi for CXRS data validation and B.Scott and F.Jenko for fruitful discussions.

\section{References}

[1] Hirsch M. et al 2001 Plasma Phys. Control. Fusion 431641 
[2] Conway G.D. et al, 2004 Plasma Phys. Control. Fusion 46951

[3] Angioni C. et al 2005 Phys. Plasmas 12040701

[4] Weisen H. et al 2005 Nucl. Fusion 45 L1

[5] Fujisawa A. et al 2000 Phys. Plasmas 74152

[6] Yoshinuma M. et al 2004 Plasma Phys. Control. Fusion 461021

[7] Cupido L., Sanchez J. and Estrada E. 2004 Rev. Sci. Instrum. 753865

[8] Silva A. et al 1999 Rev. Sci. Instrum. 701072

[9] Liewer P.C. 1985 Nucl. Fusion 25543

[10] Wootton A.J. et al 1990 Phys. Fluids B 22879

[11] Garbet X., Laurent L., Roubin J.-P. and Samain A. 1991 Nucl. Fusion 31967

[12] Rowan W.L. et al 1997 24th EPS Conf. Plasma Phys. Contr. Fusion (Berchtesgaden) (Mulhouse: EPS) ECA 21A pt.III 1193

[13] Endler M. et al 1995 Nucl. Fusion 351307

[14] Scott B. 2003 Plasma Phys. Control. Fusion 45 A385

[15] Horton W., Hu B., Dong J.Q. and Zhu P 2003 New J. Phys. 514

[16] Horton W. 1999 Rev Mod. Phys. 71735

[17] Scott B 2001 Low frequency fluid drift turbulence in magnetised plasmas Report IPP 5/92, MaxPlanck-Institut für Plasmaphysik, Garching, March 2001

[18] Connor J.W. and Wilson H.R. 1994 Plasma Phys. Control. Fusion 36719

[19] Beer M. 1995 Gyrofluid models of turbulent transport in tokamaks. Ph.D. Thesis Princeton Univ.

[20] Jenko F. 2005 Private communication Max-Planck-Institut für Plasmaphysik, Garching.

[21] Bravenec R.V. et al 2002 29th EPS Conf. Plasma Phys. Contr. Fusion (Montreux) (Mulhouse: EPS) ECA 26B P-2.067

[22] Conway G.D., Scott B., Schirmer J. et al 2005 Plasma Phys. Control. Fusion 471165

[23] Diamond P.H., Itoh S-I., Itoh K. and Hahm T.S. 2005 Plasma Phys. Control. Fusion 47 R35

[24] Garbet X. et al 2004 Plasma Phys. Control. Fusion 46 B557

[25] Poli E., Peeters A.G. and Pereverzev G.V. 2001 Comput. Phys. Commun. 13690

[26] Bulanin V.V. and Efanov M.V. 2006 Plasma Phys. Rep. 3247

[27] Gusakov E.Z., Surkov A.V. and Popov A.Yu. 2005 Plasma Phys. Control. Fusion 47959

[28] Kim J., Burrell K.H., Gohil P., Groebner R.J., Kim Y-B. et al, 1994 Phys. Rev. Lett. 722199

[29] Ida K. 1998 Plasma Phys. Control. Fusion 401429

[30] Ryter F. et al 2005 Phys. Rev. Lett. 95085001

[31] Kotschenreuther M. et al 1995 Comput. Phys. Commun. 88128

[32] Meister H. et al 2001 Nucl. Fusion 411633

[33] Burrell K.H. et al 1994 Phys. Plasmas 11536

[34] Ida K. et al 1992 Phys. Fluids B 42552

[35] Ida K. 1998 et al 1996 Plasma Phys. Control. Fusion 381433

[36] Ida K. et al 2001 Phys. Plasmas 81

[37] Peeters A.G. 2000 Phys. Plasmas 7268

[38] Hirshman S.P. and Sigmar D.J. 1981 Nucl. Fusion 211079

[39] Houlberg W.A., Shaing K.C., Hirshman S.P. and Zarnstorff M.C 1997 Phys. Plasmas 43230

[40] Kim Y.B., Diamond P.H. and Groebner R.J. 1991 Phys. Fluids B3 2050

[41] Soloman W.M. et al 2004 20th IAEA Fusion Eng. Conf. (Vilamoura) (Vienna:IAEA) IAEA-CN116/EX/P4-10 and http://www-naweb.iaea.org/napc/physics/fec/fec2004/datasets/index.html

[42] Pereverzev G.V. and Yushmanov P.N. 2002 ASTRA automated system for transport analysis in a tokamak Report IPP 5/98, Max-Planck-Institut für Plasmaphysik, Garching

[43] Crombe K. et al 2005 Poloidal rotation dynamics, radial electric field and neoclassical theory in the JET ITB region. Submitted to Phys. Rev. Lett.

[44] Rogister A.L. et al 2002 Nucl. Fusion 421144

[45] Diamond P.H. and Kim Y.B. 1991 Phys. Fluids B 31626

[46] Brower D.L. et al 1987 Phys. Rev. Lett. 5948 